Swiss Finance Institute

Research Paper Series N 09 - 49

\title{
Evolutionary Finance and Dynamic Games
}

Rabah A M IR

U niversity of A rizona

I gor V. Evstigneev

U niversity of $\mathrm{M}$ anchester

Thorsten $\mathrm{H}$ ens

U niversity of Zurich and Swiss Finance Institute

LeXU

U niversity of $\mathrm{M}$ anchester 
Established at the initiative of the Swiss Bankers' Association, the Swiss Finance Institute is a private foundation funded by the Swiss banks and SWX. It merges 3 existing foundations: the International Center FAME, the Swiss Banking School and the Stiftung "Banking and Finance" in Zurich. With its university partners, the Swiss Finance Institute pursues the objective of forming a competence center in banking and finance commensurate to the importance of the Swiss financial center. It will be active in research, doctoral training and executive education while also proposing activities fostering interactions between academia and the industry. The Swiss Finance Institute supports and promotes promising research projects in selected subject areas. It develops its activity in complete symbiosis with the NCCR FinRisk.

The National Centre of Competence in Research "Financial Valuation and Risk Management" (FinRisk) was launched in 2001 by the Swiss National Science Foundation (SNSF). FinRisk constitutes an academic forum that fosters cutting-edge finance research, education of highly qualified finance specialists at the doctoral level and knowledge transfer between finance academics and practitioners. It is managed from the University of Zurich and includes various academic institutions from Geneva, Lausanne, Lugano, St.Gallen and Zurich. For more information see www.nccr-finrisk.ch .

This paper can be downloaded without charge from the Swiss Finance Institute Research Paper Series hosted on the Social Science Research Network electronic library at:

http://ssrn.com/abstract=1536724 


\section{EVOLUTIONARY FINANCE AND DYNAMIC GAMES*}

Rabah $\operatorname{Amir}^{a}$, Igor V. Evstigneev ${ }^{b}$, Thorsten Hens $^{c}$ and Le Xu ${ }^{b}$

The paper examines a game-theoretic evolutionary model of an asset market with endogenous equilibrium asset prices. Assets pay dividends that are partially consumed and partially reinvested. The investors use general, adaptive strategies (portfolio rules), distributing their wealth between assets, depending on the exogenous states of the world and the observed history of the game. The main goal is to identify strategies, allowing an investor to "survive," i.e. to possess a positive, bounded away from zero, share of market wealth over the whole infinite time horizon. This work brings together recent studies on evolutionary finance with the classical topic of non-cooperative market games.

KEYWORDS: evolutionary finance, dynamic games, stochastic games, survival strategies

JeL Classification: C73, D52, G11

*Financial support by the Swiss National Center of Competence in Research "Financial Valuation and Risk Management" (NCCR FINRISK) is gratefully acknowledged.

${ }^{a}$ Department of Economics, University of Arizona, Tucson, AZ 857210108, U.S.A. E-mail: ramir@eller.arizona.edu. (Corresponding author.)

${ }^{b}$ Economics, School of Social Sciences, University of Manchester, Manchester M13 9PL, United Kingdom.

${ }^{c}$ Swiss Institute of Banking, University of Zurich, Plattenstrasse 32, 8032 Zurich, Switzerland. 


\section{INTRODUCTION}

1.1. The focus of the study. In this paper we examine a gametheoretic dynamic model of an asset market with endogenous equilibrium asset prices. The evolution of the market is determined by the dynamic interaction of the strategies of investors. The investors use general, adaptive strategies (portfolio rules), distributing their wealth between assets in given proportions depending on the observed exogenous random factors and the history of the market. Randomness is modeled in terms of a discrete-time stochastic process of "states of the world" with a given probability distribution. Assets pay dividends depending on the realization of this process. The dividends together with capital gains form traders' budgets, which are partially consumed and partially reinvested. A strategy profile of investors determines the process of market dynamics with equilibrium asset prices derived from a short run equilibrium of supply and demand. This random dynamical system generates a path of the unfolding simultaneous-move $N$ player stochastic game, which results in an outcome of the game characterized by a sequence of time-dependent market shares (fractions of total wealth) of each of the traders.

The main goal of the study is to identify strategies allowing an investor to "survive" in the market selection process, i.e. to maintain a positive, bounded away from zero, share of total wealth over the infinite time horizon, irrespective of the portfolio rules used by the other traders. We construct a strategy, generalizing Kelly's (1956) well-known portfolio rule of "betting your beliefs", which possesses this remarkable property of unconditional survival. Moreover, we show that the strategy possessing this property is essentially unique: any other strategy of this kind (belonging to a certain class) is asymptotically similar to the Kelly rule. The result on asymptotic uniqueness we obtain may be regarded as an analogue of turnpike theorems ${ }^{1}$, stating that all optimal or quasi-optimal paths of economic dynamics converge to each other in the long run.

\footnotetext{
${ }^{1}$ See, e.g., Nikaido (1968) and McKenzie (1986).
} 
1.2. Evolutionary finance. The model we consider is a game-theoretic version of the evolutionary model of a financial market with long-lived dividendpaying assets developed in Evstigneev et al. (2006, 2008, 2009). The general approach underlying this direction of work is to apply evolutionary dynamics - mutation and selection - to the analysis of the long-run performance of investment strategies (portfolio rules). A stock market is understood as a heterogeneous population of frequently interacting portfolio rules in competition for market capital. The ultimate goal is to build a "Darwinian theory" of portfolio selection.

Evolutionary ideas have a long history in the social sciences going back to Malthus, who played an inspirational role for Darwin (for a review of the subject see, e.g., Hodgeson (1993). A more recent stage of development of these ideas began in the 1950s with the publications of Alchian (1950) and others. An important role in this area has been played by the studies by Arthur, Holland, LeBaron, Palmer and Taylor (1997), Farmer and Lo (1999), LeBaron, Arthur and Palmer (1999), Blume and Easley (1992), Brock, Hommes and Wagener (2005) and Lux (2009).

Although the above studies provided a starting point for our line of research, our approach to evolutionary finance is different from theirs not only in the modeling frameworks and in the specific problems analyzed, but also in the general objectives of work. In particular, we deal with models based on random dynamical systems, rather than on the conventional general equilibrium settings where agents maximize discounted sums of expected utilities. The emphasis is on finding explicit formulas for surviving portfolio rules with the view to making the theory closer to practical applications. In contrast with a number of the above-mentioned papers, we use the rigorous mathematical approach, rather than computer simulations, to justify our conclusions. Considerable efforts are aimed at obtaining results in most general situations, without imposing restrictive assumptions to simplify the analysis. This requires the consideration of models having a rich mathematical structure and exploiting advanced mathematical tools.

In our work, the principal objective of the evolutionary approach consists in developing new models that would constitute a plausible alternative to conventional general equilibrium. One of the most commonly used equilibrium frameworks is that proposed by Radner (1972) — involving agents' plans, prices and price expectations. A well-known drawback of that framework is 
the necessity of agents' "perfect foresight" to establish an equilibrium. ${ }^{2}$ In particular, the market participants have to agree on the future prices for each of the possible future realizations of the states of the world (without knowing which particular state will be realized). This approach differs radically from the one based on the present evolutionary model. Here, only historical observations and the current state of the world influence current behavior; no agreement about the future market structure is required and no coordinated actions of the agents are assumed.

Another important distinction between our approach and the conventional general equilibrium paradigm lies in the data of the model we assume to be given. We avoid using unobservable agents' characteristics such as individual utilities or subjective "beliefs". The models we deal with are robust with respect to changes in such characteristics. The individual goals of investors are described in terms of properties (survival, evolutionary stability, etc.) holding almost surely, rather than in terms of the maximization of expected utilities. We consider this, robust, modelling approach as the basis for developing a new generation of dynamic equilibrium models, that could be used for practical quantitative recommendations applicable in financial industry.

In the general economics perspective, the present class of models has its roots in the Marshallian (1949) principle of temporary equilibrium (not to be confused with a different concept of temporary equilibrium due to Hicks, Lindahl and others - see, e.g. Grandmont 1988). The ideas of Marshall were developed in the framework of mathematical models in economics by Samuelson (1947, pp. 321-323). A key hypothesis needed to study the process of market dynamics by using the Marshallian "moving equilibrium method," is the co-existence of at least two sets of economic variables changing with different speeds. Then the set of variables changing slower (in our model, the set of investors' portfolios) can be temporarily fixed, while the other (in our case, the asset prices) can be assumed to rapidly reach the unique state of short-run equilibrium. For a comprehensive analysis of this approach see Schlicht (1985); in the context of evolutionary finance this modeling principle is discussed in detail in Evstigneev et al. (2008, 2009).

1.3. Evolutionary finance and game theory. Game theory has

\footnotetext{
${ }^{2}$ For discussions of this circle of questions see Laffont (1989) and Dubey et al. (1987).
} 
become one of the main general tools in mathematics-based research in Economics and Finance. As such, the model we consider in this paper fits into the broad paradigm of the applications of noncooperative game theory to the study of market behavior and can be linked to several distinct strands of literature related to this paradigm. We discuss these links below, by stressing both the common aspects and the key differences between the present model and each of these areas.

We refer, first of all, to the strand of literature on strategic market games initiated by Shapley and Shubik (Shubik 1972, Shapley and Shubik 1977) and then developed in various directions by several authors. In particular, Sahi and Yao (1989) and Amir et al. (1990) study models that do not rely on a commodity money, and are thus closer to the present study than others. Identifying our assets with standard commodities, one round of play in the present model shares some important features with static market games of the Shapley-Shubik type. In particular, given players' strategies, the endogenous price formation rule depends only on aggregates and involves setting the value of supply equal to the value of demand for each asset, as the marketclearing condition. All flows across traders are mediated via the market clearing prices. In the way of differences, our model is more easily amenable to dynamic analysis than standard market games for a number of reasons. In particular, wealth is the only critical variable to keep track of for each agent, there is no need for a commodity money in its dual role as medium of trade and utility-bearing commodity, and one naturally avoids the usual modeling dilemma of how to deal with fiat money, given that it might introduce endof-period effects by being worthless in any last period.

The central concept in the contemporary game theory is that of Nash equilibrium. One fundamental divergence of the present work with the conventional game-theoretic ideology lies in our very solution concept, the notion of a survival strategy. This notion does not explicitly involve a Nash equilibrium of any kind, nor does it entail a specification of payoff functions or preference relations that rational players in the market game would aim to maximize. What matters here is only the ability of a portfolio rule to guarantee a uniformly strictly positive share of market wealth in the long run, i.e. survival. As we emphasized above, a characteristic feature of this approach is that it relies only on model primitives that are observable and can be estimated empirically. This makes the theory closer to reality, where typically 
quantitative information about investors' utilities is lacking. In addition, investors' strategic behavior often is not fully expressible in terms of the maximization of the utility of consumption. Rather, it may involve satisficing, simple rules of thumb based on experience, and other behavioral notions. Moreover, investors might have goals not directly reducible to consumption (winning in competition, dominating a market segment, etc.).

Our notion of a survival strategy links the present work to the classical studies of games of survival pioneered by Milnor and Shapley (1957). ${ }^{3}$ These zero-sum games represent a natural two-player generalization of the classical gambler's ruin problem (Dubins and Savage, 1965). The focus is on Nash equilibria (or minmax/maxmin strategies) that are defined in terms of the probabilities of survival, which is understood in that context, in contrast with this paper, as avoiding bankruptcy at a random (finite) moment of time.

As our solution concept relies solely on the notion of long-run survival, it places emphasis on the fitness of players' strategies, rather than on players' payoffs or preferences. As such, it is somewhat reminiscent of various notions of evolutionary stable strategies in evolutionary game theory, including the celebrated concept by Maynard Smith (1982), asymptotically stable steady states of replicator dynamics processes (Samuelson, 1997), and others. However, no notions or results of the conventional evolutionary game theory are directly employed in this work. In spite of the similar general focus on issues of survival and extinction in selection processes, there is a crucial distinction between our approach and the aforementioned concepts. The latter are typically based on a given static game and random matching in a population of players, in terms of which an evolutionary process leading to survival or extinction of its participants is defined. Our notion of survival is defined in the original terms of the dynamic game describing wealth accumulation of investors, which makes it possible to address directly those questions that are of interest in the context of the modeling of asset market dynamics.

The structure of the paper is as follows. Section 2 describes the model. Section 3 states the main results (Theorems 1 and 2). Section 4 outlines a general plan of the proof of the results. The Appendix contains technical details of the proofs.

\footnotetext{
${ }^{3}$ See also Luce and Raiffa (1989, section A8.4) and Maitra and Sudderth (1996, section $7.16)$.
} 


\section{THE MODEL}

2.1. Asset market. We consider a market where $K \geq 2$ assets are traded. The market is influenced by random factors modeled in terms of an exogenous stochastic process $s_{1}, s_{2}, \ldots$, where $s_{t}$ is a random element of a measurable space $S_{t}$. At each date $t=1,2, \ldots$ assets $k=1,2, \ldots, K$ pay dividends $D_{t, k}\left(s^{t}\right) \geq 0$ depending on the history

$$
s^{t}:=\left(s_{1}, \ldots, s_{t}\right)
$$

of states of the world up to date $t$. The functions $D_{t, k}\left(s^{t}\right)$ are measurable and satisfy

$$
\sum_{k=1}^{K} D_{t, k}\left(s^{t}\right)>0 \text { for all } t, s^{t} .
$$

This condition means that at each date in each random situation at least one asset yields a strictly positive dividend. The total amount (the number of units) of asset $k$ available in the market at date $t$ is $V_{t, k}\left(s^{t}\right)>0$. For $t=0$, $V_{t, k}$ is a constant number, and for $t \geq 1, V_{t, k}\left(s^{t}\right)$ is a measurable function of $s^{t}$.

We denote by $p_{t} \in \mathbb{R}_{+}^{K}$ the vector of market prices of the assets. For each $k=1, \ldots, K$, the coordinate $p_{t, k}$ of $p_{t}=\left(p_{t, 1}, \ldots, p_{t, K}\right)$ stands for the price of one unit of asset $k$ at date $t$. There are $N \geq 2$ investors (traders) acting in the market. A portfolio of investor $i$ at date $t=0,1, \ldots$ is specified by a vector $x_{t}^{i}=\left(x_{t, 1}^{i}, \ldots, x_{t, K}^{i}\right) \in \mathbb{R}_{+}^{K}$ where $x_{t, k}^{i}$ is the amount (the number of units) of asset $k$ in the portfolio $x_{t}^{i}$. The scalar product $\left\langle p_{t}, x_{t}^{i}\right\rangle=\sum_{k=1}^{K} p_{t, k} x_{t, k}^{i}$ expresses the value of the investor $i$ 's portfolio $x_{t}^{i}$ at date $t$ in terms of the prices $p_{t, k}$. The state of the market at each date $t$ is characterized by the set of vectors $\left(p_{t}, x_{t}^{1}, \ldots, x_{t}^{N}\right)$, where $p_{t}$ is the price vector and $x_{t}^{1}, \ldots, x_{t}^{N}$ are the traders' portfolios.

At date $t=0$ the investors have initial endowments $w_{0}^{i}>0(i=$ $1,2, \ldots, N)$ that form their budgets at date 0 . Investor $i$ 's budget at date $t \geq 1$ is $\left\langle D_{t}\left(s^{t}\right)+p_{t}, x_{t-1}^{i}\right\rangle$, where $D_{t}\left(s^{t}\right):=\left(D_{t, 1}\left(s^{t}\right), \ldots, D_{t, K}\left(s^{t}\right)\right)$. It consists of two components: the dividends $\left\langle D_{t}\left(s^{t}\right), x_{t-1}^{i}\right\rangle$ paid by the portfolio $x_{t-1}^{i}$ and the market value $\left\langle p_{t}, x_{t-1}^{i}\right\rangle$ of the portfolio $x_{t-1}^{i}$ expressed in terms of the today's prices $p_{t}$. A fraction $\alpha_{t}=\alpha_{t}\left(s^{t}\right)$ of the budget is invested into assets. 
We will assume that the investment rate $0<\alpha_{t}\left(s^{t}\right)<1$ is the same for all the traders, although it may depend on time and random factors. The number $1-\alpha_{t}$ can represent the tax rate or the consumption rate. The assumption that $1-\alpha_{t}$ is the same for all the investors is quite natural in the former case. In the latter case it is indispensable since we focus in this work on the analysis of the comparative performance of trading strategies (portfolio rules) in the long run. Without this assumption, an analysis of this kind does not make sense: a seemingly worse performance of a portfolio rule in the long run might be simply due to a higher consumption rate of the investor.

We shall suppose that the function $\alpha_{t}\left(s^{t}\right)$ is measurable (for $t=0$ it is constant) and satisfies the following condition:

$$
\alpha_{t}\left(s^{t}\right)<V_{t, k}\left(s^{t}\right) / V_{t-1, k}\left(s^{t-1}\right) .
$$

This condition holds, in particular, when the total mass $V_{t, k}\left(s^{t}\right)$ of each asset $k$ does not decrease, i.e. when the right-hand side of (2) is not less than one. But (2) does not exclude the situation when $V_{t, k}$ decreases at some rate, not faster than $\alpha_{t}$.

2.2. Investment strategies. For each $t \geq 0$, every trader $i=1,2, \ldots, N$ selects a vector of investment proportions $\lambda_{t}^{i}=\left(\lambda_{t, 1}^{i}, \ldots, \lambda_{t, K}^{i}\right)$ according to which he/she plans to distribute the available budget between assets. Vectors $\lambda_{t}^{i}$ belong to the unit simplex

$$
\Delta^{K}:=\left\{\left(a_{1}, \ldots, a_{K}\right) \geq 0: a_{1}+\ldots+a_{K}=1\right\} .
$$

In terms of the game we deal with, the vectors $\lambda_{t}^{i}$ represent the players' (investors') actions or control variables. The investment proportions at each date $t \geq 0$ are selected by the $N$ traders simultaneously and independently, so that we deal here with a simultaneous-move $N$-person dynamic game. For $t \geq 1$, players' actions might depend, generally, on the history $s^{t}=$ $\left(s_{1}, \ldots, s_{t}\right)$ of the process of states of the world and the history of the game $\left(p^{t-1}, x^{t-1}, \lambda^{t-1}\right)$, where $p^{t-1}=\left(p_{0}, \ldots, p_{t-1}\right)$ is the sequence of asset price vectors up to time $t-1$, and

$$
\begin{aligned}
x^{t-1}:=\left(x_{0}, x_{1}, \ldots, x_{t-1}\right), x_{l} & =\left(x_{l}^{1}, \ldots, x_{l}^{N}\right), \\
\lambda^{t-1} & =\left(\lambda_{0}, \lambda_{1}, \ldots, \lambda_{t-1}\right), \lambda_{l}=\left(\lambda_{l}^{1}, \ldots, \lambda_{l}^{N}\right),
\end{aligned}
$$


are the sets of vectors describing the portfolios and the investment proportions of all the traders at all the dates up to $t-1$. The history of the game contains information about the market history - the sequence $\left(p_{0}, x_{0}\right), \ldots$, $\left(p_{t-1}, x_{t-1}\right)$ of the states of the market - and about the actions $\lambda_{l}^{i}$ of all the players (investors) $i=1, \ldots, N$ at all the dates $l=0, \ldots, t-1$. A vector $\Lambda_{0}^{i} \in \Delta^{K}$ and a sequence of measurable functions with values in $\Delta^{K}$

$$
\Lambda_{t}^{i}\left(s^{t}, p^{t-1}, x^{t-1}, \lambda^{t-1}\right), t=1,2, \ldots
$$

form an investment (trading) strategy $\Lambda^{i}$ of trader $i$, specifying a portfolio rule according to which trader $i$ selects investment proportions at each date $t \geq 0$. This is a general game-theoretic definition of a strategy, assuming full information about the history of the game, including the players' previous actions, and the knowledge of all the past and present states of the world.

Among general portfolio rules, we will distinguish those for which $\Lambda_{t}^{i}$ depends only on $s^{t}$, and not on the market history $\left(p^{t-1}, x^{t-1}, \lambda^{t-1}\right)$. We will call such portfolio rules basic. They play an important role in the present work: the survival strategy we construct belongs to this class.

2.3. Dynamic equilibrium. Suppose that at date 0 each investor $i$ has selected some investment proportions $\lambda_{0}^{i}=\left(\lambda_{0,1}^{i}, \ldots, \lambda_{0, K}^{i}\right) \in \Delta^{K}$. Then the amount invested in asset $k$ by trader $i$ is $\alpha_{0} \lambda_{0, k}^{i} w_{0}^{i}$ and the total amount invested in asset $k$ is $\alpha_{0} \sum_{i=1}^{N} \lambda_{0, k}^{i} w_{0}^{i}$. It is assumed that the market is always in equilibrium (asset supply is equal to asset demand), which makes it possible to determine the equilibrium price $p_{0, k}$ of each asset $k$ from the equations

$$
p_{0, k} V_{0, k}=\alpha_{0} \sum_{i=1}^{N} \lambda_{0, k}^{i} w_{0}^{i}, k=1,2, \ldots, K .
$$

On the left-hand side of (3) we have the total value $p_{0, k} V_{0, k}$ of all the assets of the type $k$ in the market (recall that the amount of each asset $k$ at date 0 is $\left.V_{0, k}\right)$. The right-hand side represents the total wealth invested in asset $k$ by all the investors. Equilibrium implies the equality in (3). The investment proportions $\lambda_{0}^{i}=\left(\lambda_{0,1}^{i}, \ldots, \lambda_{0, K}^{i}\right)$ chosen by the traders at date 0 determine their portfolios $x_{0}^{i}=\left(x_{0,1}^{i}, \ldots, x_{0, K}^{i}\right)$ at date 0 by the formula

$$
x_{0, k}^{i}=\frac{\alpha_{0} \lambda_{0, k}^{i} w_{0}^{i}}{p_{0, k}}, k=1,2, \ldots, K, i=1, \ldots, N .
$$


This formula states that the current market value $p_{0, k} x_{0, k}^{i}$ of the $k$ th position of the portfolio $x_{0}^{i}$ of investor $i$ is equal to the fraction $\lambda_{0, k}^{i}$ of the $i$ 's investment budget $\alpha_{0} w_{0}^{i}$.

Assume now that all the investors have chosen their investment proportion vectors $\lambda_{t}^{i}=\left(\lambda_{t, 1}^{i}, \ldots, \lambda_{t, K}^{i}\right)$ at date $t \geq 1$. Then the equilibrium of asset supply and demand determines the market clearing prices

$$
p_{t, k} V_{t, k}=\alpha_{t} \sum_{i=1}^{N} \lambda_{t, k}^{i}\left\langle D_{t}\left(s^{t}\right)+p_{t}, x_{t-1}^{i}\right\rangle, k=1, \ldots, K .
$$

The investment budgets $\alpha_{t}\left\langle D_{t}\left(s^{t}\right)+p_{t}, x_{t-1}^{i}\right\rangle$ of the traders $i=1,2, \ldots, N$ are distributed between assets in the proportions $\lambda_{t, k}^{i}$, so that the $k$ th position of the trader $i$ 's portfolio $x_{t}^{i}=\left(x_{t, 1}^{i}, \ldots, x_{t, K}^{i}\right)$ is

$$
x_{t, k}^{i}=\frac{\alpha_{t} \lambda_{t, k}^{i}\left\langle D_{t}\left(s^{t}\right)+p_{t}, x_{t-1}^{i}\right\rangle}{p_{t, k}}, k=1, \ldots, K, i=1, \ldots, N .
$$

Note that the price vector $p_{t}$ is determined implicitly as the solution to the system of equations (5). It can be shown that under assumption (2) a nonnegative vector $p_{t}$ satisfying these equations exists and is unique (for any $s^{t}$ and any feasible $x_{t-1}^{i}$ and $\lambda_{t}^{i}$ )- see Proposition 1 in Section 4 .

Given a strategy profile $\left(\Lambda^{1}, \ldots, \Lambda^{N}\right)$ of investors and their initial endowments $w_{0}^{1}, \ldots, w_{0}^{N}$, we can generate a path of the market game by setting

$$
\begin{gathered}
\lambda_{0}^{i}=\Lambda_{0}^{i}, i=1, \ldots, N, \\
\lambda_{t}^{i}=\Lambda_{t}^{i}\left(s^{t}, p^{t-1}, x^{t-1}, \lambda^{t-1}\right), t=1,2, \ldots, i=1, \ldots, N,
\end{gathered}
$$

and by defining $p_{t}$ and $x_{t}^{i}$ recursively according equations (3)-(6). The random dynamical system described defines step by step the vectors of investment proportions $\lambda_{t}^{i}\left(s^{t}\right)$, the equilibrium prices $p_{t}\left(s^{t}\right)$ and the investors' portfolios $x_{t}^{i}\left(s^{t}\right)$ as measurable vector functions of $s^{t}$ for each moment of time $t \geq 0$ (for $t=0$ these vectors are constant). Thus we obtain a random path of the game

$$
\left(p_{t}\left(s^{t}\right) ; x_{t}^{1}\left(s^{t}\right), \ldots, x_{t}^{N}\left(s^{t}\right) ; \lambda_{t}^{1}\left(s^{t}\right), \ldots, \lambda_{t}^{N}\left(s^{t}\right)\right),
$$

as a vector stochastic process in $\mathbb{R}_{+}^{K} \times \mathbb{R}_{+}^{K N} \times \mathbb{R}_{+}^{K N}$.

The above description of asset market dynamics requires clarification. Equations (4) and (6) make sense only if $p_{t, k}>0$, or equivalently, if the 
aggregate demand for each asset (under the equilibrium prices) is strictly positive. Those strategy profiles which guarantee that the recursive procedure described above leads at each step to strictly positive equilibrium prices will be called admissible. In what follows, we will deal only with such strategy profiles. The hypothesis of admissibility guarantees that the random dynamical system under consideration is well-defined. Under this hypothesis, we obtain by induction that on the equilibrium path all the portfolios $x_{t}^{i}=\left(x_{t, 1}^{i}, \ldots, x_{t, K}^{i}\right)$ are non-zero and the wealth

$$
w_{t}^{i}:=\left\langle D_{t}+p_{t}, x_{t-1}^{i}\right\rangle
$$

of each investor is strictly positive. Further, by summing up equations (6) over $i=1, \ldots, N$, we find that

$$
\sum_{i=1}^{N} x_{t, k}^{i}=\frac{\sum_{i=1}^{N} \alpha_{t} \lambda_{t, k}^{i}\left\langle D_{t}+p_{t}, x_{t-1}^{i}\right\rangle}{p_{t, k}}=\frac{p_{t, k} V_{t, k}}{p_{t, k}}=V_{t, k}
$$

(the market clears) for every asset $k$ and each date $t \geq 1$. The analogous relations for $t=0$ can be obtained by summing up equations (4). Thus for every equilibrium state of the market $\left(p_{t}, x_{t}^{1}, \ldots, x_{t}^{N}\right)$, we have $p_{t}>0, x_{t}^{i} \neq 0$ and (11).

We give a simple sufficient condition for a strategy profile to be admissible. This condition will hold for all the strategy profiles we shall deal with in the present paper, and in this sense it does not restrict generality. Suppose that some trader, say trader 1, uses a portfolio rule that always prescribes to invest into all the assets in strictly positive proportions $\lambda_{t, k}^{1}$. Then a strategy profile containing this portfolio rule is admissible. Indeed, for $t=0$, we get from (3) that $p_{0, k} \geq \alpha_{0} V_{0, k}^{-1} \lambda_{0, k}^{1} w_{0}^{1}>0$ and from (4) that $x_{0}^{1}=\left(x_{0,1}^{1}, \ldots, x_{0, K}^{1}\right)>0$ (coordinatewise). Assuming that $x_{t-1}^{1}>0$ and arguing by induction, we obtain $\left\langle D_{t}+p_{t}, x_{t-1}^{1}\right\rangle \geq\left\langle D_{t}, x_{t-1}^{1}\right\rangle>0$ in view of $(1)$, which in turn yields $p_{t}>0$ and $x_{t}^{1}>0$ by virtue of (5) and (6), as long as $\lambda_{t, k}^{1}>0$.

2.4. Comments on the model. An essential feature of the model we consider is the description of trading strategies in terms of investment proportions. This setting reflects the principle of active portfolio management (antipodal to a passive, buy-and-hold strategy). In this framework, even the "most passive" strategy - prescribing asset allocation in constant, time-independent, proportions - requires a periodic rebalancing of the portfolio with the view to adjusting the weights of different assets in accordance 
with changing relative prices. Although this modeling approach is in sharp contrast with the conventional general equilibrium framework, where portfolio positions are typically specified in terms of "physical units" of assets (see e.g. Magill and Quinzii 1996), it prevails in quantitative finance theory and especially in financial practice. Recommendations that fund managers or commercial finance software provide are stated in terms of investment proportions, or portfolio weights; they give an answer to the question what fraction of your wealth to allocate to one asset or another, rather than how many units of each asset to buy. Of course in a static setting when the prices are known, these two questions are equivalent, which is not the case in a dynamic framework, especially when the mechanism of asset price formation is endogenous.

The most commonly used method of quantitative fund management prescribes to select investment proportions, specifying the structure of asset allocation, and then to maintain these proportions over a certain time period. ${ }^{4}$ In our setting, these proportions are maintained during each of the time periods $(t-1, t]$. In practice, an investor who has decided to keep a certain asset allocation structure rebalances his/her portfolio on a periodic basis, say monthly, or when a substantial deviation (exceeding some fixed percentage) from the given proportions occurs owing to changes in asset prices. Then the investor sells those assets which are overweighted in the portfolio and buys those which are underweighted, and thus restores the initial balance. This process is assumed to lead in our model to a short-run equilibrium over each time period $(t-1, t]$.

The model does not describe in detail how the above process goes within each of the time periods $(t-1, t]$ (or their subperiods, when the portfolio rebalancing takes place). The generality of our framework makes it possible to admit a whole spectrum of mechanisms leading to an equilibrium in a shortrun. In reality, various auction-type mechanisms are used for the purpose of equilibrating bids and offers, resulting in market clearing. For example, the major European stock exchanges Euronext (Paris) and Xetra (Frankfurt) use computerised systems relying upon batch auctions - see, e.g., Hoffmann and van Bommel (2009). An analysis of several types of such mechanisms and their implications for the structure of trading in financial markets is

\footnotetext{
${ }^{4}$ See, e.g., Dempster et al. (2009).
} 
performed by Bottazzi et al. (2005).

The design of asset allocation structures requires an appropriate technical analysis. Theoretical studies trying to explain "Why you should rebalance your portfolio" (as Beutow et. al. entitled their 2002 paper) basically conclude that a "disciplined rebalancing strategy can add value"; see also Michaud (1998) and Plaxco and Arnott (2002). The finance literature derives this conclusion, basically, from heuristics and empirical analysis. A rigorous mathematical theory revealing the role of volatility in the endogenous growth of fixed-mix rebalancing strategies is developed in Dempster et al. (2007).

The language of investment proportions provides a formal setting most suitable for the specification of asset allocations and portfolio rebalancing. At the same time, this approach has its own drawbacks. One of those is the difficulty of the representation of some portfolio rules that are quite naturally defined in terms of "physical units" of assets (e.g. the buy-and-hold strategy) in the framework of investment proportions. Since the mechanism of price formation in our model is endogenous and all the players are market makers, such a representation might require the knowledge of the portfolio weights of all the investors, that are selected simultaneously and independently. However, both empirical evidence and theory (see the above references) suggest that we do not lose much in this connection. Simple theoretical arguments show that in a volatile market, the buy-and-hold strategy is quite often inferior to any completely diversified constant-proportions strategy involving periodic portfolio rebalancing - see Dempster et al. (2008). Paradoxically, it may happen that any strategy in the former class exhibits a negative growth rate, while the growth rate of some strategies in the latter class is positive. In our setting, the buy-and-hold strategy, if implemented, would be driven out of the market by increasing numbers of assets (e.g. when $\gamma_{t} \geq \gamma>1$, see (13) below), irrespective of the dynamics of their financial values.

The model at hand, in its present form, does not aim at comparing the performance of active and passive investment strategies. Its purpose is different: to reflect - in quantitative terms - the process of active trading characteristic for contemporary financial industry and to develop a framework more suitable in the present context than the conventional general equilibrium theory. Extensions of the model focusing on other theoretical and applied questions will constitute the subject of further research. 


\section{THE MAIN RESULTS}

3.1. The notion of survival. Let $\left(\Lambda^{1}, \ldots, \Lambda^{N}\right)$ be an admissible strategy profile of the investors. Consider the path (9) of the random dynamical system generated by this strategy profile and the given initial endowments. Let $w_{t}^{i}>0$ denote the investor $i$ 's wealth available at date $t \geq 0$. If $t=0$, then $w_{0}^{i}$ is a constant number, the initial endowment of investor $i$. If $t \geq 1$, then $w_{t}^{i}=w_{t}^{i}\left(s^{t}\right)$ is a measurable function of $s^{t}$ given by formula (10). As we have noted above, $w_{t}^{i}\left(s^{t}\right)>0$.

We are primarily interested in the long-run behavior of the relative wealth or the market shares $r_{t}^{i}:=w_{t}^{i} / W_{t}$ of the traders, where $W_{t}:=\sum_{i=1}^{N} w_{t}^{i}$ is the total market wealth. We shall say that the portfolio rule $\Lambda^{1}$ (or investor 1 using it) survives with probability one if $\inf _{t \geq 0} r_{t}^{1}>0$ almost surely (a.s.). This means that for almost all realizations of the process of states of the world $s_{1}, s_{2}, \ldots$, the market share of the first investor is bounded away from zero by a strictly positive random constant. Alternatively, we can define survival by the requirement that $\liminf _{t \rightarrow \infty} r_{t}^{1}>0$, which is equivalent, as long as the numbers $r_{t}^{1}$ are strictly positive, to the condition that inf $f_{t \geq 0} r_{t}^{1}>0$.

Let us say that a portfolio rule $\Lambda^{1}$ is a survival strategy if investor 1 using it survives with probability one regardless of what portfolio rules are used by the other investors.

We can reformulate the notion of a survival strategy in terms of the wealth processes $w_{t}^{i}(i=1,2, \ldots, N)$. Survival of a portfolio rule $\Lambda^{1}$ used by investor 1 means that $w_{t}^{1} \geq c \sum_{i=1}^{N} w_{t}^{i}$ (a.s.), where $c$ is a strictly positive random constant. The last inequality holds if and only if

$$
w_{t}^{i} \leq C w_{t}^{1}, i=1, \ldots, N, \text { (a.s.), }
$$

where $C$ is some random constant. Property (12) expresses the fact that the wealth of any investor $i$ using any strategy $\Lambda^{i}$ cannot grow asymptotically faster than the wealth of investor 1 who uses the strategy $\Lambda^{1}$. Thus, we can say that the portfolio rule $\Lambda^{1}$ is competitive: it cannot be beaten (in terms of the asymptotic growth rate of wealth) in competition with any set of strategies used by the investor 1's rivals.

3.2. The Kelly rule. Assume that the total mass of each asset grows (or decreases) at the same rate $\gamma_{t}=\gamma_{t}\left(s^{t}\right)>0$ :

$$
V_{t, k} / V_{t-1, k}=\gamma_{t}(t \geq 1) \text {. }
$$


Thus

$$
V_{t, k}=\gamma_{t} \ldots \gamma_{1} V_{k},
$$

where $V_{k}>0(k=1,2, \ldots, K)$ are the initial amounts of the assets. In the case of real dividend-paying assets - involving long-term investments in the real economy (e.g., real estate, transportation, media, infrastructure, etc.)the above assumption means that the economic system under consideration is on a balanced growth path.

Define the relative dividends of the assets $k=1, \ldots, K$ by

$$
R_{t, k}=R_{t, k}\left(s^{t}\right):=\frac{D_{t, k}\left(s^{t}\right) V_{t-1, k}\left(s^{t-1}\right)}{\sum_{m=1}^{K} D_{t, m}\left(s^{t}\right) V_{t-1, m}\left(s^{t-1}\right)}, k=1, \ldots, K, t \geq 1,
$$

and put $R_{t}\left(s^{t}\right)=\left(R_{t, 1}\left(s^{t}\right), \ldots, R_{t, K}\left(s^{t}\right)\right)$. By virtue of (14), we have

$$
R_{t, k}\left(s^{t}\right)=\frac{D_{t, k}\left(s^{t}\right) V_{k}}{\sum_{m=1}^{K} D_{t, m}\left(s^{t}\right) V_{m}} .
$$

Further, define

$$
\begin{gathered}
\rho_{t}:=\alpha_{t} / \gamma_{t}, \\
1-\rho_{t+l}, \\
\rho_{t}^{l}:=\left\{\begin{array}{cc}
\text { if } l=1, \\
\rho_{t}^{l}:=\rho_{t+1} \rho_{t+2 \ldots} \ldots \rho_{t+l-1}\left(1-\rho_{t+l}\right), & \text { if } l>1,
\end{array}\right.
\end{gathered}
$$

and assume that

$$
\rho_{t}<1-\kappa,
$$

where $\kappa$ is a strictly positive constant. Consider the portfolio rule $\Lambda^{*}$ with the vectors of investment proportions $\lambda_{t}^{*}\left(s^{t}\right)=\left(\lambda_{t, 1}^{*}\left(s^{t}\right), \ldots, \lambda_{t, K}^{*}\left(s^{t}\right)\right)$ given by

$$
\lambda_{t, k}^{*}=E_{t} \sum_{l=1}^{\infty} \rho_{t}^{l} R_{t+l, k},
$$

where $E_{t}(\cdot)=E\left(\cdot \mid s^{t}\right)$ is the conditional expectation given $s^{t}$. If $t=0$, then $E_{t}(\cdot)=E_{0}(\cdot)$ stands for the unconditional expectation $E(\cdot)$. In view of (17), the series of random variables

$$
\sum_{l=1}^{\infty} \rho_{t}^{l}=\left(1-\rho_{t+1}\right)+\rho_{t+1}\left(1-\rho_{t+2}\right)+\rho_{t+1} \rho_{t+2}\left(1-\rho_{t+3}\right)+\ldots
$$


converges uniformly, and its sum is equal to one. Therefore the series of random vectors $\sum_{l=1}^{\infty} \rho_{t}^{l} R_{t+l, k}$ in (18) converges uniformly to a random vector belonging the unit simplex $\Delta^{K}$, and so $\lambda_{t, k}^{*}$ is well-defined.

The portfolio rule specified by (18) prescribes to distribute wealth across assets in accordance with the proportions of the expected flow of their discounted future relative dividends. The discount factors $\rho_{t}^{l}$ are defined in terms of the investment rate $\alpha_{t}$ and the growth rate $\gamma_{t}$ according to formula (16). It should be emphasized that the investment proportions $\lambda_{t, k}^{*}\left(s^{t}\right)$ prescribed by the portfolio rule $\Lambda^{*}$ generally depend on time $t$ and the sequence of exogenous states of the world $s^{t}=\left(s_{1}, \ldots, s_{t}\right)$, but do not depend on the history of the game $\left(p^{t-1}, x^{t-1}, \lambda^{t-1}\right)$, so that the strategy $\Lambda^{*}$ is basic.

The strategy $\Lambda^{*}$ is a generalization of the Kelly portfolio rule of "betting your beliefs" playing an important role in capital growth theory - see Kelly (1956), Breiman (1961), Algoet and Cover (1988), and Hakansson and Ziemba (1995). If $\rho_{t}=\rho$ is constant, then formula (18) can be written as

$$
\lambda_{t, k}^{*}=E_{t} \sum_{l=1}^{\infty}\left[(1-\rho) \rho^{l-1} R_{t+l, k}\right] .
$$

Further, if the random elements $s_{t}$ are independent and identically distributed (i.i.d.) and the relative dividends $R_{t, k}\left(s^{t}\right)=R_{k}\left(s_{t}\right)$ depend only on the current state $s_{t}$ and do not explicitly depend on $t$, then $E_{t} R_{k}\left(s_{t+l}\right)=E R_{k}\left(s_{t}\right)$ $(l \geq 1)$, and so

$$
\lambda_{t, k}^{*}=E R_{k}\left(s_{t}\right),
$$

which means that the strategy $\Lambda^{*}$ is formed by the sequence of constant vectors $\left(E R_{1}\left(s_{t}\right), \ldots, E R_{K}\left(s_{t}\right)\right)$ (independent of $t$ and $\left.s^{t}\right)$. Note that in this special case, the formula (20) for $\Lambda^{*}$ does not involve the factor $\rho$. In this case, the "beliefs" at each date $t$ are concerned simply with the expected relative dividends (which do not depend on $t$ ), while in the general case, and even when $\rho$ is constant, one has to take into account the expected discounted sum of all the relative dividends at all the future dates after $t$.

3.3. The Kelly rule is a survival strategy. Assume that for all $k$ and $t$,

$$
E_{t} \sum_{l=1}^{\infty} \rho_{t}^{l} R_{t+l, k}>0 \text { (a.s.). }
$$


According to this assumption, the conditional expectation involved in the definition of the investment proportions $\lambda_{t, k}^{*}$ (see (18)) is strictly positive with probability one. Therefore we can select a version $\lambda_{t, k}^{*}\left(s^{t}\right)$ of this conditional expectation that is strictly positive for all $s^{t}$. This version will be used in the definition of the strategy $\Lambda^{*}=\left(\lambda_{t}^{*}\right)$.

A central result is as follows.

\section{THEOREM 1: The portfolio rule $\Lambda^{*}$ is a survival strategy.}

This theorem extends to the setting of long-lived dividend-paying assets an analogous result established in the framework of a model with one-period, "short-lived" assets in Amir et al. (2008). That framework, studied also by Blume and Easley (1992) and Amir et al. (2005), may be regarded as a limiting case as $\rho \rightarrow 0$ (with constant $\rho_{t}=\rho$ ) of the one considered in the present paper. In the case of short-lived assets, the version of the Kelly rule that turns out to be a survival strategy is defined by the investment proportions $\lambda_{t, k}^{*}=E_{t} R_{t+1, k}$. These proportions are limits of those in (19) as $\rho \rightarrow 0$. The analysis of the model with long-lived assets in which general strategies are allowed and no assumptions on the process of states of the world are imposed is much more demanding. It requires a substantial generalization of the concept of the Kelly portfolio rule, involving the discounting of the future dividends, and it is based on new techniques (relying upon stochastic Lyapunov functions) designed for the analysis of random dynamical systems arising in connection with the dynamic market games at hand.

As we have noted above, if the states of the world $s_{t}$ are i.i.d. and the functions $R_{t, k}\left(s^{t}\right)=R_{k}\left(s_{t}\right)$ depend only on $s_{t}$ and do not explicitly depend on $t$, then the investment proportions $\lambda_{k}^{*}=E R_{k}\left(s_{t}\right)$ of the strategy $\Lambda^{*}$ are constant: they depend neither on time nor on the states of the world (such strategies are called simple). A version of the asset market model with longlived assets in which all the investors use only simple portfolio rules and the states of the world are i.i.d. is considered in Evstigneev et al. (2008). It is shown in that context that the strategy $\Lambda^{*}$ not only survives, but outperforms all other simple strategies. Those investors who use $\Lambda^{*}$ dominate the market, i.e. gather in the limit total market wealth, while those who use simple strategies distinct from $\Lambda^{*}$ vanish: their market shares tend to zero with probability one. This is not so in the model considered in the present paper, 
where general, not necessarily simple, portfolio rules are allowed. Here, $\Lambda^{*}$ investors survive, i.e. keep market shares bounded away from zero a.s., but they do not necessarily dominate the market.

3.4. Asymptotic uniqueness of the survival strategy. As we have already noted, the portfolio rule $\Lambda^{*}$ belongs to the class of basic portfolio rules: the investment proportions $\lambda_{t}^{*}\left(s^{t}\right)$ depend only on the history $s^{t}$ of the process of states of the world, and do not depend on the market history. The following theorem shows that in this class the survival strategy $\Lambda^{*}=\left(\lambda_{t}^{*}\right)$ is essentially unique: any other basic survival strategy is asymptotically similar to $\Lambda^{*}$.

TheOREm 2. If $\Lambda=\left(\lambda_{t}\right)$ is a basic survival strategy, then

$$
\sum_{t=0}^{\infty}\left\|\lambda_{t}^{*}-\lambda_{t}\right\|^{2}<\infty \text { (a.s.). }
$$

Here, we denote by $\|\cdot\|$ the Euclidean norm in a finite-dimensional space. Theorem 2 is akin to various turnpike results in the theory of economic dynamics, expressing the idea that all optimal or asymptotically optimal paths of an economic system follow in the long run essentially the same route: the turnpike (Nikaido 1968, McKenzie 1986). Survival strategies $\Lambda$ can be characterized by the property that the wealth $w_{t}^{j}$ of any investor $j$ cannot grow infinitely faster (with strictly positive probability) than the wealth of investor $i$ using $\Lambda$. The class of such investment strategies is similar to the class of "good" paths of economic dynamics, as introduced by Gale (1967) - paths that cannot be "infinitely worse" than the turnpike. Theorem 2 is a direct analogue of Gale's turnpike theorem for good paths (Gale, 1967, Theorem 8); for a stochastic version of this result see Arkin and Evstigneev (1987, Chapter 4, Theorem 6).

Note that the class of basic strategies is sufficient in the following sense. Any sequence of vectors $r_{t}=\left(r_{t}^{1}, \ldots, r_{t}^{N}\right)\left(r_{t}=r_{t}\left(s^{t}\right)\right)$ of market shares generated by some strategy profile $\left(\Lambda^{1}, \ldots, \Lambda^{N}\right)$ can be generated by a strategy profile $\left(\lambda_{t}^{1}\left(s^{t}\right), \ldots, \lambda_{t}^{N}\left(s^{t}\right)\right)$ consisting of basic portfolio rules. The corresponding vector functions $\lambda_{t}^{i}\left(s^{t}\right)$ can be defined recursively by (7) and (8), using (3)-(6). Thus it is sufficient to prove Theorem 1 only for basic portfolio rules; this will imply that the portfolio rule (18) survives in competition with 
any, not necessarily basic, strategies. Such considerations cannot be automatically applied to the problem of asymptotic characterization of general survival strategies. This problem remains open; it indicates an interesting direction for further research.

\section{MATHEMATICAL ANALYSIS OF THE MODEL}

4.1. Some auxiliary propositions. In this section we set out the program of proving Theorems 1 and 2. The proofs are divided into several steps which are described in several propositions and lemmas below. Based on these auxiliary results, we present at the end of the section the final steps of the proofs of Theorems 1 and 2. Technical details of the derivation of the auxiliary results are relegated to the Appendix.

The first proposition establishes the existence and uniqueness of an equilibrium price vector at each date $t \geq 0$.

Proposition 1: Let assumption (2) hold. Let $x_{t-1}=\left(x_{t-1}^{1}, \ldots, x_{t-1}^{N}\right)$ be a set of vectors $x_{t-1}^{i} \in \mathbb{R}_{+}^{K}$ satisfying (11). Then for any $s^{t}$ there exists a unique solution $p_{t} \in \mathbb{R}_{+}^{K}$ to equations (5). This solution is measurable with respect to all the parameters involved in (5).

In the next proposition, we derive a system of equations governing the dynamics of the market shares of the investors given their admissible strategy profile $\left(\Lambda^{1}, \ldots, \Lambda^{N}\right)$. Consider the path (9) of the random dynamical system generated by $\left(\Lambda^{1}, \ldots, \Lambda^{N}\right)$ and the sequence of vectors $r_{t}=\left(r_{t}^{1}, \ldots, r_{t}^{N}\right)$, where $r_{t}^{i}$ is the investor $i$ 's market share at date $t$.

Proposition 2: The following equations hold:

$$
r_{t+1}^{i}=\sum_{k=1}^{K}\left[\rho_{t+1}\left\langle\lambda_{t+1, k}, r_{t+1}\right\rangle+\left(1-\rho_{t+1}\right) R_{t+1, k}\right] \frac{\lambda_{t, k}^{i} r_{t}^{i}}{\left\langle\lambda_{t, k}, r_{t}\right\rangle},
$$

$i=1, \ldots, N, t \geq 0$.

The next proposition shows that it is sufficient to prove Theorem 1 when $N=2$, i.e., the general model can be reduced to the case of two investors. Define

$$
\tilde{\lambda}_{t, k}^{2}=\frac{\lambda_{t, k}^{2} r_{t}^{2}+\ldots+\lambda_{t, k}^{N} r_{t}^{N}}{1-r_{t}^{1}}
$$


Note that $1-r_{t}^{1}=r_{t}^{2}+\ldots+r_{t}^{N}>0$, and so $\tilde{\lambda}_{t, k}^{2}$ is well-defined. Furthermore

$$
\sum_{k=1}^{K} \tilde{\lambda}_{t, k}^{2}=\frac{r_{t}^{2}+\ldots+r_{t}^{N}}{1-r_{t}^{1}}=1
$$

which means that the vector $\tilde{\lambda}_{t}^{2}:=\left(\tilde{\lambda}_{t, 1}^{2}, \ldots, \tilde{\lambda}_{t, K}^{2}\right)$ belongs to the unit simplex $\Delta^{K}$. Thus the sequence of vectors $\tilde{\lambda}_{t}^{2}=\tilde{\lambda}_{t}^{2}\left(s^{t}\right)$ defines a portfolio rule, which will be denoted by $\tilde{\Lambda}$. Define

$$
\tilde{r}_{t}^{1}=r_{t}^{1}, \tilde{r}_{t}^{2}=1-r_{t}^{1}, \tilde{r}_{t}=\left(\tilde{r}_{t}^{1}, \tilde{r}_{t}^{2}\right), \tilde{\lambda}_{t, k}^{1}=\lambda_{t, k}^{1}, \tilde{\lambda}_{t, k}=\left(\tilde{\lambda}_{t, k}^{1}, \tilde{\lambda}_{t, k}^{2}\right)
$$

Proposition 3: We have

$$
\tilde{r}_{t+1}^{i}=\sum_{k=1}^{K}\left[\rho_{t+1}\left\langle\tilde{\lambda}_{t+1, k}, \tilde{r}_{t+1}\right\rangle+\left(1-\rho_{t+1}\right) R_{t+1, k}\right] \frac{\tilde{\lambda}_{t, k}^{i} \tilde{r}_{t}^{i}}{\left\langle\tilde{\lambda}_{t, k}, \tilde{r}_{t}\right\rangle}, i=1,2, t \geq 0 .
$$

Thus in the model with two investors $i=1,2$ using the strategies $\Lambda$ and $\tilde{\Lambda}$, respectively, the market share $\tilde{r}_{t}^{1}$ of the first investor coincides with $r_{t}^{1}$ (coming from the original model) and the market share $\tilde{r}_{t}^{2}$ of the second is equal to $1-r_{t}^{1}$.

Consider the model with two traders $(N=2)$ using strategies $\Lambda^{i}=$ $\left(\lambda_{t, k}^{i}\left(s^{t}\right)\right), i=1,2$, and denote by $z_{t}$ the ratio $r_{t}^{1} / r_{t}^{2}$ of their market shares.

Proposition 4: The process $z_{t}$ is governed by the following random $d y$ namical system:

$$
z_{t+1}=z_{t} \frac{\sum_{k=1}^{K}\left[\rho_{t+1} \lambda_{t+1, k}^{2}+\left(1-\rho_{t+1}\right) R_{t+1, k}\right] \frac{\lambda_{t, k}^{1}}{\lambda_{t, k}^{1} z_{t}+\lambda_{t, k}^{2}}}{\sum_{k=1}^{K}\left[\rho_{t+1} \lambda_{t+1, k}^{1}+\left(1-\rho_{t+1}\right) R_{t+1, k}\right] \frac{\lambda_{t, k}^{2}}{\lambda_{t, k}^{1} z_{t}+\lambda_{t, k}^{2}}} .
$$

In the next proposition, we derive an equation which can be used as an equivalent definition of the portfolio rule $\Lambda^{*}$. 
Proposition 5: The portfolio rule $\Lambda^{*}=\left(\lambda_{t, k}^{*}\right)$ satisfies

$$
E_{t}\left[\rho_{t+1} \lambda_{t+1, k}^{*}+\left(1-\rho_{t+1}\right) R_{t+1, k}\right]=\lambda_{t, k}^{*} \text { (a.s.). }
$$

It can be shown (by using a contraction principle) that $\Lambda^{*}$ is a unique solution to (26), but this fact will not be needed in what follows.

4.2. The plan of the proof of Theorem 1 is as follows. Proposition 3 shows that we can consider, without loss of generality, the case of two investors. This reduces the dimension of the original random dynamical system from a general $N$ to $N=2$. Proposition 4 describes a one-dimensional system which governs the evolution of the ratio $z_{t}=r_{t}^{1} / r_{t}^{2}$ of the market shares of the two investors, and thus reduces the dimension of the problem to 1 . Our goal is to show that the random sequence $\left(z_{t}\right)$ defined recursively by (25) is bounded away from zero almost surely. To this end it turns out to be convenient to take a "step back" and to increase the dimension to $K$ (the number of assets). Assuming that the first trader uses the investment proportions $\lambda_{t, k}^{1}=\lambda_{t, k}^{*}\left(s^{t}\right)$ prescribed by the portfolio rule $\Lambda^{*}$ and the second trader employs investment proportions $\lambda_{t, k}^{2}=\lambda_{t, k}\left(s^{t}\right)$ specified by some other portfolio rule $\Lambda$, we introduce the following change of variables

$$
y_{t}^{k}=\lambda_{t, k} / z_{t}, \quad k=1, \ldots, K,
$$

and define $y_{t}:=\left(y_{t}^{1}, \ldots, y_{t}^{K}\right)$. We examine the dynamics of the random vectors $y_{t}=y_{t}\left(s^{t}\right)$ implied by the system (25). The norm $\left|y_{t}\right|:=\sum_{k}\left|y_{t}^{k}\right|$ of the vector $y_{t} \geq 0$ is equal to $\sum_{k}\left(\lambda_{t, k} / z_{t}\right)=1 / z_{t}$, and what we need is to show that $1 /\left|y_{t}\right|$ is bounded away from zero a.s.. To prove this, we construct a stochastic Lyapunov function - a function of $y_{t}$ which forms a non-negative supermartingale $\left(\zeta_{t}\right)$ along a path $\left(y_{t}\right)$ of the system at hand (see Lemma 3 below). By using the supermartingale convergence theorem, we prove that the stochastic process $\zeta_{t}$ converges a.s., which implies that it is bounded a.s.. We complete the proof by showing that the boundedness of $\zeta_{t}$ implies that $z_{t}=1 /\left|y_{t}\right|$ is bounded away from zero.

4.3. Some inequalities and supermartingale properties. We begin the realization of the above plan with two lemmas containing inequalities involving the variables $y_{t}^{k}$ defined by (27). Define the non-negative random variables

$$
Y_{t}:=\ln \left(1+\left|y_{t}\right|\right)=-\ln r_{t}^{1},
$$




$$
Z_{t, k}:=\ln \left(1+\frac{y_{t}^{k}}{\lambda_{t, k}^{*}}\right)=\ln \left(1+\frac{r_{t}^{2} \lambda_{t, k}}{r_{t}^{1} \lambda_{t, k}^{*}}\right), Z_{t}:=\sum_{k=1}^{K} \lambda_{t, k}^{*} Z_{t, k},
$$

and put

$$
U_{t}:=Y_{t}-Z_{t}
$$

LEMMA 1: The following inequality holds:

$$
\rho_{t+1} Z_{t+1}+\left(1-\rho_{t+1}\right) Y_{t+1} \leq \sum_{k=1}^{K}\left[\rho_{t+1} \lambda_{t+1, k}^{*}+\left(1-\rho_{t+1}\right) R_{t+1, k}\right] Z_{t, k}
$$

LEMMA 2: We have

$$
U_{t}=\sum_{k=1}^{K} \lambda_{t, k}^{*} \ln \frac{\lambda_{t, k}^{*}}{r_{t}^{1} \lambda_{t, k}^{*}+r_{t}^{2} \lambda_{t, k}} \geq 0 .
$$

From the above results, we derive the following fact.

LEMMA 3: The random sequence

$$
\zeta_{t}:=\rho_{t} Z_{t}+\left(1-\rho_{t}\right) Y_{t}
$$

is a non-negative supermartingale satisfying

$$
\zeta_{t}-E_{t} \zeta_{t+1} \geq\left(1-\rho_{t}\right) U_{t}
$$

The following two lemmas will be used in proof of Theorem 2 .

LEMMA 4. Let $\zeta_{t}$ be a supermartingale such that $\inf _{t} E \zeta_{t}>-\infty$. Then the series of non-negative random variables $\sum_{t=0}^{\infty}\left(\zeta_{t}-E_{t} \zeta_{t+1}\right)$ converges a.s.

LEMMA 5. For any vectors $\left(a_{1}, \ldots, a_{K}\right)>0$ and $\left(b_{1}, \ldots, b_{K}\right) \geq 0$ satisfying $\sum a_{k}=\sum b_{k}=1$, the following inequality holds

$$
\sum_{k=1}^{K} a_{k} \ln a_{k}-\sum_{k=1}^{K} a_{k} \ln b_{k} \geq \frac{1}{4} \sum_{k=1}^{K}\left(a_{k}-b_{k}\right)^{2} .
$$


4.4. Proof of Theorem 1. Since $\zeta_{t}$ is a non-negative supermartingale, the sequence $\zeta_{t}$ converges a.s., and hence it is bounded above a.s. by some random constant $C$. This implies (see (33) and (17)) that $\left(1-\rho_{t}\right) Y_{t} \leq \zeta_{t} \leq C$ (a.s.), and so

$$
-\ln r_{t}^{1}=Y_{t} \leq \zeta_{t} /\left(1-\rho_{t}\right) \leq B \text { (a.s.), }
$$

where $B:=C / \kappa$. Therefore $r_{t}^{1} \geq e^{-B}$ (a.s.).

4.5. Proof of Theorem 2. Let $\Lambda=\left(\lambda_{t}\right)$ be a basic survival strategy. Suppose that investors $i=1,2, \ldots, N-1$ use the strategy $\Lambda^{*}=\left(\lambda_{t}^{*}\right)$ and investor $N$ uses $\Lambda$. By summing up equations (23) with $\lambda_{t}^{i}=\lambda_{t}^{*}$ over $i=$ $1, \ldots, N-1$, we obtain

$$
\begin{gathered}
\hat{r}_{t+1}^{1}=\sum_{k=1}^{K}\left[\rho_{t+1}\left(\lambda_{t+1, k}^{*} \hat{r}_{t+1}^{1}+\lambda_{t+1, k}\left(1-\hat{r}_{t+1}^{1}\right)\right)+\right. \\
\left.\quad\left(1-\rho_{t+1}\right) R_{t+1, k}\right] \frac{\lambda_{t, k}^{*} \hat{r}_{t}^{1}}{\lambda_{t, k}^{*} \hat{r}_{t}^{1}+\lambda_{t, k}\left(1-\hat{r}_{t}^{1}\right)} .
\end{gathered}
$$

where $\hat{r}_{t}^{1}:=r_{t}^{1}+\ldots+r_{t}^{N-1}$ is the market share of the group of investors $i=1,2, \ldots, N-1$ and $1-\hat{r}_{t}=r_{t}^{N}$ is the market share of investor $N$. We used here the fact that

$$
\begin{gathered}
\left\langle\lambda_{t, k}, r_{t}\right\rangle=\sum_{i=1}^{N} \lambda_{t, k}^{i} r_{t}^{i}=\sum_{i=1}^{N-1} \lambda_{t, k}^{*} r_{t}^{i}+\lambda_{t, k} r_{t}^{N}= \\
\lambda_{t, k}^{*} \sum_{i=1}^{N-1} r_{t}^{i}+\lambda_{t, k} r_{t}^{N}=\lambda_{t, k}^{*} \hat{r}_{t}^{1}+\lambda_{t, k}\left(1-\hat{r}_{t}^{1}\right) .
\end{gathered}
$$

Further, we have

$$
\begin{gathered}
1-\hat{r}_{t+1}^{1}=\sum_{k=1}^{K}\left[\rho_{t+1}\left(\lambda_{t+1, k}^{*} \hat{r}_{t+1}^{1}+\lambda_{t+1, k}\left(1-\hat{r}_{t+1}^{1}\right)\right)+\right. \\
\left.\left(1-\rho_{t+1}\right) R_{t+1, k}\right] \frac{\lambda_{t, k}\left(1-\hat{r}_{t}^{1}\right)}{\lambda_{t, k}^{*} \hat{r}_{t}^{1}+\lambda_{t, k}\left(1-\hat{r}_{t}^{1}\right)} .
\end{gathered}
$$


Thus the dynamics of the market shares $\hat{r}_{t}^{1}=r_{t}^{1}+\ldots+r_{t}^{N-1}, 1-\hat{r}_{t}^{1}=r_{t}^{N}$ is exactly the same as the dynamics of the market shares $\hat{r}_{t}^{1}, \hat{r}_{t}^{2}=1-\hat{r}_{t}^{1}$ of two investors $i=1,2(N=2)$ using the strategies $\left(\lambda_{t}^{1}\right)=\left(\lambda_{t}^{*}\right)$ and $\left(\lambda_{t}^{2}\right)=\left(\lambda_{t}\right)$, respectively. Since $\left(\lambda_{t}\right)$ is a survival strategy, the random sequence $r_{t}^{N}=$ $1-\hat{r}_{t}^{1}=\hat{r}_{t}^{2}$ is bounded away from zero almost surely.

Since investor 1 uses the strategy $\Lambda^{*}$, by virtue of Lemma 3 the sequence $\zeta_{t}$ defined by (33) is a non-negative supermartingale, and inequality (34) holds. In view of Lemma 4 , the series $\sum_{t=0}^{\infty}\left(\zeta_{t}-E_{t} \zeta_{t+1}\right)$ of non-negative random variables converges a.s. The inequality

$$
\zeta_{t}-E_{t} \zeta_{t+1} \geq\left(1-\rho_{t}\right) \sum_{k=1}^{K} \lambda_{t, k}^{*} \ln \frac{\lambda_{t, k}^{*}}{\hat{r}_{t}^{1} \lambda_{t, k}^{*}+\hat{r}_{t}^{2} \lambda_{t, k}}
$$

established in Lemmas 2 and 3 and assumption (17) imply that

$$
\sum_{t=0}^{\infty} \sum_{k=1}^{K} \lambda_{t, k}^{*} \ln \frac{\lambda_{t, k}^{*}}{\hat{r}_{t}^{1} \lambda_{t, k}^{*}+\hat{r}_{t}^{2} \lambda_{t, k}}<\infty \text { (a.s.). }
$$

Finally, we observe that

$$
\begin{gathered}
\sum_{k=1}^{K} \lambda_{t, k}^{*} \ln \frac{\lambda_{t, k}^{*}}{\hat{r}_{t}^{1} \lambda_{t, k}^{*}+\hat{r}_{t}^{2} \lambda_{t, k}}= \\
\sum_{k=1}^{K} \lambda_{t, k}^{*} \ln \lambda_{t, k}^{*}-\sum_{k=1}^{K} \lambda_{t, k}^{*} \ln \left[\hat{r}_{t}^{1} \lambda_{t, k}^{*}+\hat{r}_{t}^{2} \lambda_{t, k}\right] \geq \\
\frac{1}{4} \sum_{k=1}^{K}\left[\lambda_{t, k}^{*}-\left(1-\hat{r}_{t}^{2}\right) \lambda_{t, k}^{*}-\hat{r}_{t}^{2} \lambda_{t, k}\right]^{2}=\frac{1}{4} \sum_{k=1}^{K}\left(\hat{r}_{t}^{2} \lambda_{t, k}^{*}-\hat{r}_{t}^{2} \lambda_{t, k}\right)^{2}= \\
\frac{1}{4}\left(\hat{r}_{t}^{2}\right)^{2} \sum_{k=1}^{K}\left(\lambda_{t, k}^{*}-\lambda_{t, k}\right)^{2}=\frac{1}{4}\left(\hat{r}_{t}^{2}\right)^{2}\left\|\lambda_{t}^{*}-\lambda_{t}\right\|^{2},
\end{gathered}
$$

(see (35)), where the sequence $\hat{r}_{t}^{2}$ is bounded away from zero a.s., as long as $\left(\lambda_{t}\right)$ is a survival strategy. Therefore

$$
\hat{r}_{t}^{2} \geq c>0 \text { (a.s.), }
$$

where $c$ is a random constant. From relations (36)-(38) we conclude that the series $\sum_{t=0}^{\infty}\left\|\lambda_{t}^{*}-\lambda_{t}\right\|^{2}$ converges a.s., which completes the proof of Theorem 2. 


\section{APPENDIX}

Proof of Proposition 1: Fix some $t$ and $s^{t}$ and consider the operator transforming a vector $p=\left(p_{1}, \ldots, p_{K}\right) \in \mathbb{R}_{+}^{K}$ into the vector $q=\left(q_{1}, \ldots, q_{K}\right) \in$ $\mathbb{R}_{+}^{K}$ with coordinates

$$
q_{k}=\alpha_{t} V_{t, k}^{-1} \sum_{i=1}^{N} \lambda_{t, k}^{i}\left\langle D_{t}+p, x_{t-1}^{i}\right\rangle .
$$

This operator is contracting in the norm $\|p\|_{V}:=\sum_{k}\left|p_{k}\right| V_{t-1, k}$. Indeed, by virtue of (2) we have

$$
\tilde{\alpha}:=\max _{k=1, \ldots, K}\left\{\alpha_{t} V_{t-1, k} V_{t, k}^{-1}\right\}<1,
$$

and so

$$
\begin{gathered}
\left\|q-q^{\prime}\right\|_{V}=\sum_{k=1}^{K}\left|q_{k}-q_{k}^{\prime}\right| V_{t-1, k} \leq \\
\alpha_{t} \sum_{k=1}^{K} V_{t-1, k} V_{t, k}^{-1} \sum_{i=1}^{N} \lambda_{t, k}^{i}\left|\left\langle p-p^{\prime}, x_{t-1}^{i}\right\rangle\right| \leq \tilde{\alpha} \sum_{i=1}^{N} \sum_{k=1}^{K} \lambda_{t, k}^{i}\left|\left\langle p-p^{\prime}, x_{t-1}^{i}\right\rangle\right|= \\
\tilde{\alpha} \sum_{i=1}^{N}\left|\left\langle p-p^{\prime}, x_{t-1}^{i}\right\rangle\right| \leq \tilde{\alpha} \sum_{i=1}^{N} \sum_{m=1}^{K}\left|p_{m}-p_{m}^{\prime}\right| x_{t-1, m}^{i}= \\
\tilde{\alpha} \sum_{m=1}^{K} \sum_{i=1}^{N}\left|p_{m}-p_{m}^{\prime}\right| x_{t-1, m}^{i}=\tilde{\alpha} \sum_{m=1}^{K}\left|p_{m}-p_{m}^{\prime}\right| V_{t-1, m}=\tilde{\alpha}|| p-p^{\prime} \|_{V}
\end{gathered}
$$

where the last but one equality follows from (11). By using the contraction principle, we obtain the existence, uniqueness and measurability of the solution to (5).

Proof of Proposition 2: From (5) and (6) we get

$$
\begin{gathered}
p_{t, k}=V_{t, k}^{-1} \alpha_{t} \sum_{i=1}^{N} \lambda_{t, k}^{i}\left\langle p_{t}+D_{t}, x_{t-1}^{i}\right\rangle= \\
\alpha_{t} V_{t, k}^{-1} \sum_{i=1}^{N} \lambda_{t, k}^{i} w_{t}^{i}=\alpha_{t} V_{t, k}^{-1}\left\langle\lambda_{t, k}, w_{t}\right\rangle,
\end{gathered}
$$




$$
x_{t, k}^{i}=\frac{V_{t, k} \lambda_{t, k}^{i} w_{t}^{i}}{\left\langle\lambda_{t, k}, w_{t}\right\rangle}
$$

where $t \geq 1, w_{t}:=\left(w_{t}^{1}, \ldots, w_{t}^{N}\right)$ and $\lambda_{t, k}:=\left(\lambda_{t, k}^{1}, \ldots, \lambda_{t, k}^{N}\right)$. The analogous formulas for $t=0$,

$$
p_{0, k}=\alpha_{0} V_{0, k}^{-1}\left\langle\lambda_{0, k}, w_{0}\right\rangle, x_{0, k}^{i}=\frac{V_{0, k} \lambda_{0, k}^{i} w_{0}^{i}}{\left\langle\lambda_{0, k}, w_{0}\right\rangle},
$$

follow from (3) and (4). Consequently, we have

$$
\begin{gathered}
w_{t+1}^{i}=\sum_{k=1}^{K}\left(p_{t+1, k}+D_{t+1, k}\right) x_{t, k}^{i}= \\
\sum_{k=1}^{K}\left(\alpha_{t+1} \frac{\left\langle\lambda_{t+1, k}, w_{t+1}\right\rangle}{V_{t+1, k}}+D_{t+1, k}\right) \frac{V_{t, k} \lambda_{t, k}^{i} w_{t}^{i}}{\left\langle\lambda_{t, k}, w_{t}\right\rangle}= \\
\sum_{k=1}^{K}\left(\alpha_{t+1} \frac{\left\langle\lambda_{t+1, k}, w_{t+1}\right\rangle V_{t, k}}{V_{t+1, k}}+D_{t+1, k} V_{t, k}\right) \frac{\lambda_{t, k}^{i} w_{t}^{i}}{\left\langle\lambda_{t, k}, w_{t}\right\rangle} t \geq 0 .
\end{gathered}
$$

By summing up these equations over $i=1, \ldots, N$, we obtain

$$
\begin{gathered}
W_{t+1}=\sum_{k=1}^{K}\left(\alpha_{t+1} \frac{\left\langle\lambda_{t+1, k}, w_{t+1}\right\rangle V_{t, k}}{V_{t+1, k}}+D_{t+1, k} V_{t, k}\right) \frac{\sum_{i=1}^{N} \lambda_{t, k}^{i} w_{t}^{i}}{\left\langle\lambda_{t, k}, w_{t}\right\rangle}= \\
\sum_{k=1}^{K}\left(\alpha_{t+1} \frac{\left\langle\lambda_{t+1, k}, w_{t+1}\right\rangle V_{t, k}}{V_{t+1, k}}+D_{t+1, k} V_{t, k}\right) .
\end{gathered}
$$

As long as

$$
V_{t+1, k} / V_{t, k}=\gamma_{t+1}>0
$$

(see (13)), we have

$$
\begin{gathered}
W_{t+1}=\sum_{k=1}^{K}\left(\alpha_{t+1} \gamma_{t+1}^{-1}\left\langle\lambda_{t+1, k}, w_{t+1}\right\rangle+D_{t+1, k} V_{t, k}\right)= \\
\sum_{k=1}^{K}\left(\alpha_{t+1} \gamma_{t+1}^{-1}\left\langle\lambda_{t+1, k}, w_{t+1}\right\rangle+D_{t+1, k} V_{t, k}\right)=\alpha_{t+1} \gamma_{t+1}^{-1} W_{t+1}+\sum_{k=1}^{K} D_{t+1, k} V_{t, k} .
\end{gathered}
$$


This implies the formula

$$
W_{t+1}=\frac{1}{1-\alpha_{t+1} \gamma_{t+1}^{-1}} \sum_{m=1}^{K} D_{t+1, m} V_{t, m}
$$

where $\alpha_{t+1} \gamma_{t+1}^{-1}=\rho_{t+1}$. From (42) and (43), we find

$$
w_{t+1}^{i}=\sum_{k=1}^{K}\left(\rho_{t+1}\left\langle\lambda_{t+1, k}, w_{t+1}\right\rangle+D_{t+1, k} V_{t, k}\right) \frac{\lambda_{t, k}^{i} w_{t}^{i}}{\left\langle\lambda_{t, k}, w_{t}\right\rangle} t \geq 0 .
$$

Dividing both sides of this equation by $W_{t+1}$ and using (44), we get

$$
r_{t+1}^{i}=\sum_{k=1}^{K}\left[\rho_{t+1}\left\langle\lambda_{t+1, k}, r_{t+1}\right\rangle+\left(1-\rho_{t+1}\right) \frac{D_{t+1, k} V_{t, k}}{\sum_{m=1}^{K} D_{t+1, m} V_{t, m}}\right] \frac{\lambda_{t, k}^{i} w_{t}^{i} / W_{t}}{\left\langle\lambda_{t, k}, w_{t}\right\rangle / W_{t}},
$$

which yields (23) by virtue of (13) and (15).

Proof of Proposition 3: In view of (24) and (23) we have

$$
\begin{gathered}
r_{t+1}^{1}=\sum_{k=1}^{K}\left\{\rho_{t+1}\left[\lambda_{t+1, k}^{1} r_{t+1}^{1}+\left(1-r_{t+1}^{1}\right) \tilde{\lambda}_{t+1, k}^{2}\right]+\right. \\
\left.\left(1-\rho_{t+1}\right) R_{t+1, k}\right\} \frac{\lambda_{t, k}^{1} r_{t}^{1}}{\lambda_{t, k}^{1} r_{t}^{1}+\left(1-r_{t}^{1}\right) \tilde{\lambda}_{t, k}^{2}} .
\end{gathered}
$$

By summing up equations (23) over $i=2, \ldots, N$, we find

$$
\tilde{r}_{t+1}^{2}=1-r_{t+1}^{1}=\sum_{k=1}^{K}\left[\rho_{t+1}\left\langle\lambda_{t+1, k}, r_{t+1}\right\rangle+\left(1-\rho_{t+1}\right) R_{t+1, k}\right] \frac{\left(1-r_{t}^{1}\right) \tilde{\lambda}_{t, k}^{2}}{\left\langle\lambda_{t, k}, r_{t}\right\rangle}
$$

Thus we obtain

$$
\begin{gathered}
\tilde{r}_{t+1}^{2}=\sum_{k=1}^{K}\left\{\rho_{t+1}\left[\lambda_{t+1, k}^{1} r_{t+1}^{1}+\left(1-r_{t+1}^{1}\right) \tilde{\lambda}_{t+1, k}^{2}\right]+\right. \\
\left.\left(1-\rho_{t+1}\right) R_{t+1, k}\right\} \frac{\left(1-r_{t}^{1}\right) \tilde{\lambda}_{t, k}^{2}}{\lambda_{t, k}^{1} r_{t}^{1}+\left(1-r_{t}^{1}\right) \tilde{\lambda}_{t, k}^{2}}
\end{gathered}
$$


which completes the proof.

Proof of Proposition 4: By using (23) with $N=2$, we get

$$
r_{t+1}^{i}=\sum_{k=1}^{K}\left[\rho_{t+1}\left(\lambda_{t+1, k}^{i} r_{t+1}^{i}+\lambda_{t+1, k}^{j}\left(1-r_{t+1}^{i}\right)\right)+\left(1-\rho_{t+1}\right) R_{t+1, k}\right] \frac{\lambda_{t, k}^{i} r_{t}^{i}}{\lambda_{t, k}^{i} r_{t}^{i}+\lambda_{t, k}^{j} r_{t}^{j}},
$$

where $i, j \in\{1,2\}$ and $i \neq j$. Setting $C_{t, k}^{i j}:=\lambda_{t, k}^{i} r_{t}^{i} /\left(\lambda_{t, k}^{i} r_{t}^{i}+\lambda_{t, k}^{j} r_{t}^{j}\right)$, we obtain $r_{t+1}^{i}\left[1+\rho_{t+1} \sum_{k=1}^{K}\left(\lambda_{t+1, k}^{j}-\lambda_{t+1, k}^{i}\right) C_{t, k}^{i j}\right]=\sum_{k=1}^{K}\left[\rho_{t+1} \lambda_{t+1, k}^{j}+\left(1-\rho_{t+1}\right) R_{t+1, k}\right] C_{t, k}^{i j}$.

Thus

$$
\frac{r_{t+1}^{i}}{r_{t+1}^{j}}=\frac{A_{t+1}^{i j} / B_{t+1}^{i j}}{A_{t+1}^{j i} / B_{t+1}^{j i}}
$$

where

$$
\begin{gathered}
A_{t+1}^{i j}:=\sum_{k=1}^{K}\left[\rho_{t+1} \lambda_{t+1, k}^{j}+\left(1-\rho_{t+1}\right) R_{t+1, k}\right] C_{t, k}^{i j}, \\
B_{t+1}^{i j}:=1+\rho_{t+1} \sum_{k=1}^{K}\left(\lambda_{t+1, k}^{j}-\lambda_{t+1, k}^{i}\right) C_{t, k}^{i j} .
\end{gathered}
$$

Observe that $B_{t+1}^{j i}=B_{t+1}^{i j}$. Indeed,

$$
\begin{gathered}
B_{t+1}^{i j}-B_{t+1}^{j i}=\rho_{t+1} \sum_{k=1}^{K}\left[\left(\lambda_{t+1, k}^{j}-\lambda_{t+1, k}^{i}\right) C_{t, k}^{i j}-\left(\lambda_{t+1, k}^{i}-\lambda_{t+1, k}^{j}\right) C_{t, k}^{j i}\right]= \\
\rho_{t+1} \sum_{k=1}^{K}\left(\lambda_{t+1, k}^{j}-\lambda_{t+1, k}^{i}\right)=0
\end{gathered}
$$

because $C_{t, k}^{i j}+C_{t, k}^{j i}=1$. Consequently,

$$
\frac{r_{t+1}^{1}}{r_{t+1}^{2}}=\frac{A_{t+1}^{12}}{A_{t+1}^{21}}=\frac{r_{t}^{1}}{r_{t}^{2}} \frac{\sum_{k=1}^{K}\left[\rho_{t+1} \lambda_{t+1, k}^{2}+\left(1-\rho_{t+1}\right) R_{t+1, k}\right] \frac{\lambda_{t, k}^{1}}{\lambda_{t, k}^{1} r_{t}^{1} / r_{t}^{2}+\lambda_{t, k}^{2}}}{\sum_{k=1}^{K}\left[\rho_{t+1} \lambda_{t+1, k}^{1}+\left(1-\rho_{t+1}\right) R_{t+1, k}\right] \frac{\lambda_{t, k}^{2}}{\lambda_{t, k}^{1} r_{t}^{1} / r_{t}^{2}+\lambda_{t, k}^{2}}},
$$


which yields (25).

Proof of Proposition 5: By virtue of (18), we have

$$
\begin{gathered}
E_{t}\left(\rho_{t+1} \lambda_{t+1, k}^{*}\right)=E_{t}\left(\rho_{t+1} E_{t+1} \sum_{l=1}^{\infty} \rho_{t+1}^{l} R_{t+1+l, k}\right)= \\
E_{t}\left(E_{t+1} \sum_{l=1}^{\infty} \rho_{t+1} \rho_{t+1}^{l} R_{t+1+l, k}\right)=E_{t}\left(\sum_{l=1}^{\infty} \rho_{t+1} \rho_{t+1}^{l} R_{t+1+l, k}\right),
\end{gathered}
$$

and so

$$
\begin{gathered}
E_{t}\left[\rho_{t+1} \lambda_{t+1, k}^{*}+\left(1-\rho_{t+1}\right) R_{t+1, k}\right]=E_{t}\left[\sum_{l=1}^{\infty} \rho_{t+1} \rho_{t+1}^{l} R_{t+1+l, k}+\left(1-\rho_{t+1}\right) R_{t+1, k}\right]= \\
E_{t}\left(\sum_{l=1}^{\infty} \rho_{t}^{l+1} R_{t+l+1, k}+\rho_{t}^{1} R_{t+1, k}\right)=E_{t} \sum_{l=1}^{\infty} \rho_{t}^{l} R_{t+l, k}=\lambda_{t, k}^{*}
\end{gathered}
$$

because $1-\rho_{t+1}=\rho_{t}^{1}$ and

$$
\rho_{t}^{l+1}=\rho_{t+1} \rho_{t+2 \ldots .} \rho_{t+l}\left(1-\rho_{t+l+1}\right)=\rho_{t+1} \rho_{t+1}^{l}
$$

for $l \geq 1$.

Proof of Lemma 1: From formula (25) with $\lambda_{t, k}^{1}=\lambda_{t, k}^{*}$ and $\lambda_{t, k}^{2}=\lambda_{t, k}$, we get

$$
\begin{gathered}
\sum_{k=1}^{K}\left[\rho_{t+1} \lambda_{t+1, k}^{*}+\left(1-\rho_{t+1}\right) R_{t+1, k}\right] \frac{\lambda_{t, k}}{\lambda_{t, k}^{*} z_{t}+\lambda_{t, k}}= \\
\sum_{k=1}^{K}\left[\rho_{t+1} \frac{\lambda_{t+1, k}}{z_{t+1}}+\left(1-\rho_{t+1}\right) \frac{R_{t+1, k}}{z_{t+1}}\right] \frac{\lambda_{t, k}^{*} z_{t}}{\lambda_{t, k}^{*} z_{t}+\lambda_{t, k}} .
\end{gathered}
$$

By using the notation $y_{t}^{k}=\lambda_{t, k} / z_{t}$ and the fact that $\left|y_{t}\right|=1 / z_{t}$, we write

$$
\begin{aligned}
& \sum_{k=1}^{K}\left[\rho_{t+1} \lambda_{t+1, k}^{*}+\left(1-\rho_{t+1}\right) R_{t+1, k}\right] \frac{y_{t}^{k}}{\lambda_{t, k}^{*}+y_{t}^{k}}= \\
& \sum_{k=1}^{K}\left[\rho_{t+1} y_{t+1}^{k}+\left(1-\rho_{t+1}\right) R_{t+1, k}\left|y_{t+1}\right|\right] \frac{\lambda_{t, k}^{*}}{\lambda_{t, k}^{*}+y_{t}^{k}},
\end{aligned}
$$


which implies

$$
\rho_{t+1} \sum_{k=1}^{K} \frac{\lambda_{t, k}^{*} y_{t+1}^{k}-\lambda_{t+1, k}^{*} y_{t}^{k}}{\lambda_{t, k}^{*}+y_{t}^{k}}+\left(1-\rho_{t+1}\right) \sum_{k=1}^{K} R_{t+1, k} \frac{\lambda_{t, k}^{*}\left|y_{t+1}\right|-y_{t}^{k}}{\lambda_{t, k}^{*}+y_{t}^{k}}=0 .
$$

We have

$$
\begin{gathered}
\frac{\lambda_{t, k}^{*} y_{t+1}^{k}-\lambda_{t+1, k}^{*} y_{t}^{k}}{\lambda_{t, k}^{*}+y_{t}^{k}}=\lambda_{t+1, k}^{*} \frac{y_{t+1}^{k} / \lambda_{t+1, k}^{*}-y_{t}^{k} / \lambda_{t, k}^{*}}{1+y_{t}^{k} / \lambda_{t, k}^{*}}= \\
\lambda_{t+1, k}^{*}\left(\frac{1+y_{t+1}^{k} / \lambda_{t+1, k}^{*}}{1+y_{t}^{k} / \lambda_{t, k}^{*}}-1\right) \geq \lambda_{t+1, k}^{*} \ln \frac{1+y_{t+1}^{k} / \lambda_{t+1, k}^{*}}{1+y_{t}^{k} / \lambda_{t, k}^{*}},
\end{gathered}
$$

where the last relation follows from the inequality $a-1 \geq \ln a(a>0)$. By using (46) and the same inequality, we find

$$
\begin{gathered}
\sum_{k=1}^{K} \frac{\lambda_{t, k}^{*} y_{t+1}^{k}-\lambda_{t+1, k}^{*} y_{t}^{k}}{\lambda_{t, k}^{*}+y_{t}^{k}} \geq \sum_{k=1}^{K} \lambda_{t+1, k}^{*}\left[\ln \left(1+\frac{y_{t+1}^{k}}{\lambda_{t+1, k}^{*}}\right)-\ln \left(1+\frac{y_{t}^{k}}{\lambda_{t, k}^{*}}\right)\right]= \\
\sum_{k=1}^{K} \lambda_{t+1, k}^{*}\left(Z_{t+1, k}-Z_{t, k}\right)=Z_{t+1}-\sum_{k=1}^{K} \lambda_{t+1, k}^{*} Z_{t, k} .
\end{gathered}
$$

Further, we have

$$
\begin{gathered}
\frac{\lambda_{t, k}^{*}\left|y_{t+1}\right|-y_{t}^{k}}{\lambda_{t, k}^{*}+y_{t}^{k}}=\frac{\lambda_{t, k}^{*}\left|y_{t+1}\right|+\lambda_{t, k}^{*}}{\lambda_{t, k}^{*}+y_{t}^{k}}-1 \geq \\
\ln \frac{\lambda_{t, k}^{*}\left|y_{t+1}\right|+\lambda_{t, k}^{*}}{\lambda_{t, k}^{*}+y_{t}^{k}}=\ln \frac{\left|y_{t+1}\right|+1}{1+y_{t}^{k} / \lambda_{t, k}^{*}},
\end{gathered}
$$

and so

$$
\begin{gathered}
\sum_{k=1}^{K} R_{t+1, k} \frac{\lambda_{t, k}^{*}\left|y_{t+1}\right|-y_{t}^{k}}{\lambda_{t, k}^{*}+y_{t}^{k}} \geq \sum_{k=1}^{K} R_{t+1, k} \ln \frac{1+\left|y_{t+1}\right|}{1+y_{t}^{k} / \lambda_{t, k}^{*}}= \\
\ln \left(1+\left|y_{t+1}\right|\right)-\sum_{k=1}^{K} R_{t+1, k} \ln \left(1+y_{t}^{k} / \lambda_{t, k}^{*}\right)=Y_{t+1}-\sum_{k=1}^{K} R_{t+1, k} Z_{t, k}
\end{gathered}
$$

(see (28) and (29)), which yields

$$
\sum_{k=1}^{K} R_{t+1, k} \frac{\lambda_{t, k}^{*}\left|y_{t+1}\right|-y_{t}^{k}}{\lambda_{t, k}^{*}+y_{t}^{k}} \geq Y_{t+1}-\sum_{k=1}^{K} R_{t+1, k} Z_{t, k}
$$


By combining (45), (47) and (48), we find

$$
\begin{gathered}
0 \geq \rho_{t+1}\left(Z_{t+1}-\sum_{k=1}^{K} \lambda_{t+1, k}^{*} Z_{t, k}\right)+\left(1-\rho_{t+1}\right)\left(Y_{t+1}-\sum_{k=1}^{K} R_{t+1, k} Z_{t, k}\right)= \\
\rho_{t+1} Z_{t+1}+\left(1-\rho_{t+1}\right) Y_{t+1}-\sum_{k=1}^{K}\left[\rho_{t+1} \lambda_{t+1, k}^{*}+\left(1-\rho_{t+1}\right) R_{t+1, k}\right] Z_{t, k}
\end{gathered}
$$

which proves $(31)$.

Proof of Lemma 2: To prove the first relation in (32) we proceed as follows:

$$
\begin{gathered}
U_{t}=Y_{t}-Z_{t}=-\ln r_{t}^{1}-\sum_{k=1}^{K} \lambda_{t, k}^{*} \ln \left[1+\frac{r_{t}^{2} \lambda_{t, k}}{r_{t}^{1} \lambda_{t, k}^{*}}\right]= \\
\sum_{k=1}^{K} \lambda_{t, k}^{*} \ln \frac{1}{r_{t}^{1}}+\sum_{k=1}^{K} \lambda_{t, k}^{*} \ln \frac{r_{t}^{1} \lambda_{t, k}^{*}}{r_{t}^{1} \lambda_{t, k}^{*}+r_{t}^{2} \lambda_{t, k}}=\sum_{k=1}^{K} \lambda_{t, k}^{*} \ln \frac{\lambda_{t, k}^{*}}{r_{t}^{1} \lambda_{t, k}^{*}+r_{t}^{2} \lambda_{t, k}} .
\end{gathered}
$$

The last relation in (32) follows from the elementary inequality $\sum_{k=1}^{K} a_{k} \ln a_{k}-$ $\sum_{k=1}^{K} a_{k} \ln b_{k} \geq 0$, which is presented in a somewhat refined form in Lemma 5 .

Proof of Lemma 3: It is clear that $\zeta_{t} \geq 0$. By taking the conditional expectation $E_{t}(\cdot)$ of both sides of inequality (31) and using (26), we obtain

$$
E_{t} \zeta_{t+1} \leq \sum_{k=1}^{K} Z_{t, k} E_{t}\left[\rho_{t+1} \lambda_{t+1, k}^{*}+\left(1-\rho_{t+1}\right) R_{t+1, k}\right]=\sum_{k=1}^{K} Z_{t, k} \lambda_{t, k}^{*}=Z_{t}
$$

In view of (30), we get

$$
\begin{gathered}
E_{t} \zeta_{t+1}+\left(1-\rho_{t}\right) U_{t} \leq Z_{t}+\left(1-\rho_{t}\right) Y_{t}-\left(1-\rho_{t}\right) Z_{t}= \\
\rho_{t} Z_{t}+\left(1-\rho_{t}\right) Y_{t}=\zeta_{t},
\end{gathered}
$$

which proves (34). Thus $E_{t} \zeta_{t+1} \leq \zeta_{t}-\left(1-\rho_{t}\right) U_{t} \leq \zeta_{t}$ because $U_{t} \geq 0$ (see Lemma 2). The last inequality implies $E \zeta_{t} \leq E \zeta_{0}=\zeta_{0}<+\infty$. Since $\zeta_{t} \geq 0$, we have $E\left|\zeta_{t}\right|<\infty$, and so $\zeta_{t}$ is a supermartingale. 
Proof of Lemma 4: The random variables $\eta_{t}:=\zeta_{t}-E_{t} \zeta_{t+1}$ are nonnegative by the definition of a supermartingale. Further, we have

$$
\sum_{t=0}^{T-1} E \eta_{t}=\sum_{t=0}^{T-1}\left(E \zeta_{t}-E \zeta_{t+1}\right)=E \zeta_{0}-E \zeta_{T}
$$

and so the sequence $\sum_{t=0}^{T-1} E \eta_{t}$ is bounded because $\sup _{T}\left(-E \zeta_{T}\right)=-\inf _{T} E \zeta_{T}<$ $+\infty$. Therefore the series of the expectations $\sum_{t=0}^{\infty} E \eta_{t}$ of the non-negative random variables $\eta_{t}$ converges, which implies $\sum_{t=0}^{\infty} \eta_{t}<\infty$ a.s. because $E \sum_{t=0}^{\infty} \eta_{t}=\sum_{t=0}^{\infty} E \eta_{t}$ (the last equality holds for any sequence $\eta_{t} \geq 0$ ).

Proof of Lemma 5: We have $\ln x \leq x-1$, which implies $(\ln x) / 2=$ $\ln \sqrt{x} \leq \sqrt{x}-1$, and so $-\ln x \geq 2-2 \sqrt{x}$. By using this inequality, we get

$$
\begin{gathered}
\sum_{k=1}^{K} a_{k}\left(\ln a_{k}-\ln b_{k}\right)=-\sum_{k=1}^{K} a_{k} \ln \frac{b_{k}}{a_{k}} \geq \sum_{k=1}^{K} a_{k}\left(2-2 \frac{\sqrt{b_{k}}}{\sqrt{a_{k}}}\right)= \\
2-2 \sum_{k=1}^{K} \sqrt{a_{k} b_{k}}=\sum_{k=1}^{K}\left(a_{k}-2 \sqrt{a_{k} b_{k}}+b_{k}\right)=\sum_{k=1}^{K}\left(\sqrt{a_{k}}-\sqrt{b_{k}}\right)^{2} .
\end{gathered}
$$

This yields (35) because $(\sqrt{a}-\sqrt{b})^{2} \geq(a-b)^{2} / 4$ for $0 \leq a, b \leq 1$. 


\section{REFERENCES}

Algoet, P.H., and T.M. Cover (1988): "Asymptotic optimality and asymptotic equipartition properties of log-optimum investment," Annals of Probability, 16, 876-898.

Alchian, A. (1950): "Uncertainty, evolution and economic theory," Journal of Political Economy, 58, 211-221.

Amir, R., I.V. Evstigneev, T. Hens, and K.R. Schenk-Hoppé (2005): "Market selection and survival of investment strategies," Journal of Mathematical Economics, 41 (Special Issue on Evolutionary Finance), 105-122.

Amir, R., I.V. Evstigneev, and K.R. Schenk-Hoppé (2008): "Asset market games of survival," Swiss Finance Institute, Research Paper Series No. 08-31.

Amir, R., S. Sahi, M. Shubik, and S. Yao (1990): "A strategic market game with complete markets," Journal of Economic Theory, 51, 126-143.

Arkin, V.I., and I.V. Evstigneev (1987): Stochastic Models of Control and Economic Dynamics. London: Academic Press.

Arthur, W.B., J.H. Holland, B. LeBaron, R.G. Palmer, and P. Taylor (1997): "Asset pricing under endogenous expectations in an artificial stock market," in The Economy as an Evolving Complex System, II, ed. by W.B. Arthur, S. Durlauf and D. Lane. Reading, MA: Addison Wesley, pp. 15-44.

Buetow, G., R. Sellers, D. Trotter, E. Hunt, and W.A. Whipple (2002): "The benefits of rebalancing," Journal of Portfolio Management, 28, 23-32.

Blume, L., and D. Easley (1992): "Evolution and market behavior," Journal of Economic Theory, 58, 9-40.

Bottazzi, G., G. Dosi, and I. Rebesco (2005): "Institutional architectures and behavioral ecologies in the dynamics of financial markets," Journal of Mathematical Economics, 41, 197-228.

Brock, A. W., Hommes, C. H., and F. O. O.Wagener (2005): "Evolutionary dynamics in markets with many trader types," Journal of Mathematical Economics, 41 (Special Issue on Evolutionary Finance), 7-42.

Breiman, L. (1961): "Optimal gambling systems for favorable games," Fourth Berkeley Symposium on Math. Statist. and Probability, 1. Berkeley: University of California Press, pp. 65-78.

Dempster, M.A.H., I.V. Evstigneev, and K.R. Schenk-Hoppé (2007): "Volatility-induced financial growth," Quantitative Finance, 7, 151-160. 
Dempster, M.A.H., I.V. Evstigneev, and K.R. Schenk-Hoppé (2008): "Financial markets. The joy of volatility," Quantitative Finance, 8, 1-3.

Dempster, M.A.H., G. Mitra, and G. Pflug, eds. (2009): Quantitative Fund Management. Boca Raton: Chapman and Hall / CRC Financial Mathematics Series (Taylor and Francis Group).

Dubey, P., J. Geanakoplos, and M. Shubik (1987): "The revelation of information in strategic market games. A critique of rational expectations equilibrium," Journal of Mathematical Economics, 16, 105-137.

Dubins, L., and L.M. Savage (1965): How to Gamble if You Must. New York: Mc-Graw-Hill.

Evstigneev, I.V., T. Hens, and K.R. Schenk-Hoppé (2006): "Evolutionary stable stock markets," Economic Theory, 27, 449-468.

Evstigneev, I.V., T. Hens, and K.R. Schenk-Hoppé (2008): "Globally evolutionarily stable portfolio rules," Journal of Economic Theory, 140, 197228.

Evstigneev, I.V., T. Hens, and K.R. Schenk-Hoppé (2009): "Evolutionary finance," in Handbook of Financial Markets: Dynamics and Evolution, Chapter 9, ed. by T. Hens and K.R. Schenk-Hoppé. Amsterdam: Elsevier, pp. 507-566.

Farmer, J.D., and A.W. Lo (1999): "Frontiers of finance: Evolution and efficient markets," Proceedings of the National Academy of Sciences, 96, 9991-9992.

Gale, D. (1967): "On optimal development in a multi-sector economy," Review of Economic Studies, 34, 1-18.

Grandmont, J-M., ed. (1988): Temporary Equilibrium. San Diego: Academic Press.

Hakansson, N.H., and W.T. Ziemba (1995): "Capital growth theory," Handbooks in Operations Research and Management Science, 9, Finance, ed. by R.A. Jarrow, V. Maksimovic and W.T. Ziemba. Amsterdam: Elsevier, pp. 65-86.

Hodgeson, G. M. (1993): Economics and Evolution: Bringing Life Back into Economics. Cambridge: Polity Press (Blackwell Publishers).

Hoffmann, P., and J. van Bommel (2009): Pre-trade transparency in call auctions: Quantity discovery versus price efficiency, Working paper. (Available at SSRN: http://ssrn.com/abstract=1343939.) 
Kelly, J.L. (1956): "A new interpretation of information rate," Bell System Technical Journal 35, 917-926.

Laffont, J.-J., (1989): The Economics of Uncertainty and Information. Cambridge MA: MIT Press.

LeBaron, B., W.B. Arthur, and R. Palmer (1999): "Time series properties of an artificial stock market," Journal of Economic Dynamics and Control, $23,1487-1516$.

Luce, R., and H. Raiffa (1989): Games and Decisions, 2nd Edition. New York: Dover.

Lux, T. (2009): "Stochastic behavioral asset pricing models and the stylized facts," in Handbook of Financial Markets: Dynamics and Evolution, Chapter 3, ed. by T. Hens and K.R. Schenk-Hoppé. Amsterdam: Elsevier, pp. 161-211.

Magill, M., and M. Quinzii (1996): Theory of Incomplete Markets. Cambridge MA: MIT Press.

Maitra, A., and W.D. Sudderth (1996): Discrete Gambling and Stochastic Games. New York: Springer.

Marshall, A. (1949): Principles of Economics, 8th Edition. London: Macmillan.

Maynard Smith, J. (1982): Evolution and the Theory of Games. Cambridge, UK: Cambridge University Press.

McKenzie, L.W. (1986): "Optimal economic growth, turnpike theorems and comparative dynamics", Handbook of Mathematical Economics III, ed. by K.J. Arrow and M.D. Intrilligator. Amsterdam: North Holland, pp. 12811355.

Michaud, R.O. (1998): Efficient Asset Management. Boston, MA: Harvard Business School Press.

Milnor, J., and L.S. Shapley (1957): "On games of survival", Contributions to the Theory of Games III, ed. by M. Dresher et al. Princeton: Princeton University Press, pp. 15-45.

Nikaido, H. (1968): Convex Structures and Economic Theory. New York: Academic Press.

Plaxco, L.M., and R.D. Arnott (2002): "Rebalancing a global policy benchmark", Journal of Portfolio Management, 28, 9-22.

Radner, R. (1972): "Existence of equilibrium of plans, prices, and price expectations in a sequence of markets," Econometrica, 40, 289-303. 
Sahi, S., and S. Yao (1989): "The noncooperative equilibria of a trading economy with complete markets and consistent prices," Journal of Mathematical Economics, 18, 325-346.

Samuelson, L. (1997): Evolutionary Games and Equilibrium Selection. Cambridge, MA: MIT Press.

Samuelson, P.A. (1947): Foundations of Economic Analysis. Cambridge, MA: Harvard University Press.

Schlicht, E. (1985): Isolation and Aggregation in Economics. Berlin: Springer.

Secchi, P., and W.D. Sudderth (2001): "Stay-in-a-set games," International Journal of Game Theory, 30, 479-490.

Shapley, L.S. (1953): "Stochastic games," Proceedings of the National Academy of Sciences of the USA, 39, 1095-1100.

Shapley, L.S. (1976): "Noncooperative general exchange," Theory and Measurement of Economic Externalities. New York: Academic Press, pp. 155-175.

Shapley, L.S., and M. Shubik (1977): "Trade using one commodity as a means of payment," Journal of Political Economy, 85, 937-968.

Shubik, M. (1972): "A theory of money and financial institutions: Fiat money and noncooperative equilibrium in a closed economy," International Journal of Game Theory, 1, 243-268. 


\section{swiss : finance: institute}

c/o University of Geneva

40 bd du Pont d'Arve

1211 Geneva 4

Switzerland

$\mathrm{T}+41223798471$

F +41 223798277

RPS@sfi.ch

www.SwissFinanceInstitute.ch 